\title{
Front Matter: Volume 9767
}

, "Front Matter: Volume 9767," Proc. SPIE 9767, Novel In-Plane Semiconductor Lasers XV, 976701 (21 June 2016); doi: 10.1117/12.2239587

SPIE. Event: SPIE OPTO, 2016, San Francisco, California, United States 


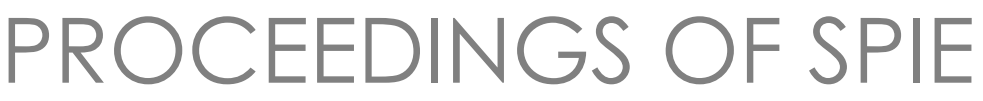

\title{
Novel In-Plane Semiconductor Lasers XV
}

\author{
Alexey A. Belyanin \\ Peter M. Smowton
}

Editors

\section{5-18 February 2016 \\ San Francisco, California, United States}

Sponsored and Published by

SPIE 
The papers in this volume were part of the technical conference cited on the cover and title page. Papers were selected and subject to review by the editors and conference program committee. Some conference presentations may not be available for publication. Additional papers and presentation recordings may be available online in the SPIE Digital Library at SPIEDigitallibrary.org.

The papers reflect the work and thoughts of the authors and are published herein as submitted. The publisher is not responsible for the validity of the information or for any outcomes resulting from reliance thereon.

Please use the following format to cite material from these proceedings:

Author(s), "Title of Paper," in Novel In-Plane Semiconductor Lasers XV, edited by Alexey A. Belyanin, Peter M. Smowton, Proceedings of SPIE Vol. 9767 (SPIE, Bellingham, WA, 2016) Six-digit Article CID Number.

ISSN: 0277-786X

ISSN: 1996-756X (electronic)

ISBN: 9781510600027

Published by

SPIE

P.O. Box 10, Bellingham, Washington 98227-0010 USA

Telephone +1 3606763290 (Pacific Time) · Fax +1 3606471445

SPIE.org

Copyright (C) 2016, Society of Photo-Optical Instrumentation Engineers.

Copying of material in this book for internal or personal use, or for the internal or personal use of specific clients, beyond the fair use provisions granted by the U.S. Copyright Law is authorized by SPIE subject to payment of copying fees. The Transactional Reporting Service base fee for this volume is $\$ 18.00$ per article (or portion thereof), which should be paid directly to the Copyright Clearance Center (CCC), 222 Rosewood Drive, Danvers, MA 01923. Payment may also be made electronically through CCC Online at copyright.com. Other copying for republication, resale, advertising or promotion, or any form of systematic or multiple reproduction of any material in this book is prohibited except with permission in writing from the publisher. The CCC fee code is 0277-786X/16/\$18.00.

Printed in the United States of America.

Publication of record for individual papers is online in the SPIE Digital Library.

\section{SPIE. DIGITAL}

Paper Numbering: Proceedings of SPIE follow an e-First publication model. A unique citation identifier (CID) number is assigned to each article at the time of publication. Utilization of CIDs allows articles to be fully citable as soon as they are published online, and connects the same identifier to all online and print versions of the publication. SPIE uses a six-digit CID article numbering system structured as follows:

- The first four digits correspond to the SPIE volume number.

- The last two digits indicate publication order within the volume using a Base 36 numbering system employing both numerals and letters. These two-number sets start with 00, 01, 02, 03, 04, $05,06,07,08,09,0 A, 0 B \ldots$ OZ, followed by 10-1Z, 20-2Z, etc. The CID Number appears on each page of the manuscript. 


\title{
Contents
}

\author{
vii Authors \\ ix Conference Committee
}

\section{STRUCTURE AND CAVITY DEVELOPMENTS}

976703 Type-I QW cascade diode lasers for spectral region above $3 \boldsymbol{\mu m}$ [9767-2]

976705 Generation of 7W nanosecond pulses with 670nm ridge-waveguide lasers [9767-4]

976706 GaAs-based self-aligned stripe superluminescent diodes processed normal to the cleaved facet [9767-5]

\section{MATERIALS DEVELOPMENTS}

976709 Microscopic modelling of opto-electronic properties of dilute bismide materials for the mid-IR [9767-8]

9767 OA First demonstration of orange-yellow light emitter devices in InGaP/InAIGaP laser structure using strain-induced quantum well intermixing technique [9767-9]

\section{BLUE/GREEN EMITTERS}

9767 OC Room-temperature continuous-wave operation of BeZnCdSe quantum-well green-toyellow laser diodes with sub-10 mA threshold current [9767-11]

9767 OE Studies on 405nm blue-violet diode laser with external grating cavity [9767-13]

9767 OF Large TE polarized optical gain from AllnN-delta-GaN quantum well for ultraviolet lasers [9767-14]

\section{QUANTUM DOTS}

$9767 \mathrm{Ol} \quad 1.5 \mu \mathrm{m}$ quantum dot laser material with high temperature stability of threshold current density and external differential efficiency [9767-17]

9767 0J High-temperature continuous wave operation (up to $100^{\circ} \mathrm{C}$ ) of InAs/InGaAs quantum dot electrically injected microdisk lasers [9767-18]

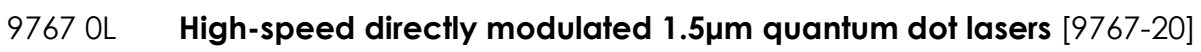


9767 ON Timing jitter performance of mode-locked external cavity multi-quantum-well semiconductor lasers [9767-22]

976700 Interaction of phase and amplitude shaping in an external cavity semiconductor laser [9767-23]

DFB AND DBRS

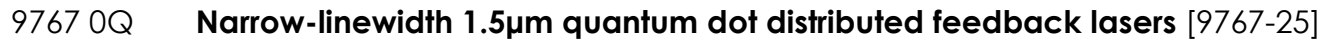

9767 OR Pulsed hybrid dual wavelength Y-branch-DFB laser-tapered amplifier system suitable for water vapor detection at $965 \mathrm{~nm}$ with $16 \mathrm{~W}$ peak power [9767-26]

9767 OS Difference frequency modulation of multi-section dual-mode lasers with nanoscale surface gratings [9767-27]

9767 OT $\quad 5,000 \mathrm{~h}$ reliable operation of $785 \mathrm{~nm}$ dual-wavelength DBR-RW diode lasers suitable for Raman spectroscopy and SERDS [9767-28]

9767 OU First demonstration of single-mode distributed feedback type-I GaSb cascade diode laser emitting near $2.9 \mu \mathrm{m}$ [9767-29]

\section{LASERS ON SILICON}

9767 OY Cavity enhanced 1.5 $\mu \mathrm{m}$ LED with silicon as a hole injector [9767-33]

976710 Photonic-crystal lasers on silicon for chip-scale optical interconnects (Invited Paper) [9767-35]

INTERBAND AND QUANTUM CASCADE LASERS

976712 Interband cascade laser sources in the mid-infrared for green photonics [9767-37]

976713 Step-taper active-region quantum cascade lasers for carrier-leakage suppression and high internal differential efficiency [9767-38]

976714 Surface-emitting quantum cascade laser with 2nd-order metal-semiconductor gratings for single-lobe emission [9767-39]

QCLS: COMBS AND MODE-LOCKING II

9767 1A Frequency comb operation of long-cavity terahertz quantum-cascade lasers (Invited Paper) [9767-45] 
9767 1E Quantum dots as active material for quantum cascade lasers: comparison to quantum wells [9767-50]

\section{HIGH-BRIGHTNESS LASERS}

$976711 \quad$ DBR tapered diode laser at $1030 \mathrm{~nm}$ with nearly diffraction-limited narrowband emission and $12.7 \mathrm{~W}$ of optical output power [9767-53]

$97671 \mathrm{~J} \mathrm{High} \mathrm{performances} \mathrm{of} \mathrm{very} \mathrm{long} \mathrm{(13.5mm)} \mathrm{tapered} \mathrm{laser} \mathrm{emitting} \mathrm{at} 975 \mathrm{~nm}$ [9767-54]

$97671 \mathrm{~K}$ Three-section master oscillator power amplifier at $1.57 \mu \mathrm{m}$ for lidar measurements of atmospheric carbon dioxide [9767-55]

9767 IL Novel approaches to increasing the brightness of broad area lasers (Invited Paper) [9767-56]

\section{QCLS: CAVITIES, INTEGRATION, AND APPLICATIONS}

9767 IR Monolithic integration of a quantum cascade laser array and an echelle grating multiplexer for widely tunable mid-infrared sources [9767-62]

9767 is Regrowth-free mid-infrared distributed feedback quantum cascade lasers with sub-watt power consumption [9767-63]

$97671 \mathrm{~T}$ Spectroscopic benzene detection using a broadband monolithic DFB-QCL array [9767-64]

$97671 \mathrm{U}$ Cascade laser applications: trends and challenges [9767-65]

9767 IV Single-mode enhancement in coupled-cavity quantum cascade lasers [9767-66]

POSTER SESSION

9767 IW Simulation of broad spectral bandwidth emitters at $1060 \mathrm{~nm}$ for optical coherence tomography [9767-67]

976721 Three-dimensional finite-difference time-domain modelling of photonic crystal surfaceemitting lasers [9767-72] 
Proc. of SPIE Vol. $9767976701-6$

Downloaded From: https://www.spiedigitallibrary.org/conference-proceedings-of-spie on 26 Apr 2023 Terms of Use: https://www.spiedigitallibrary.org/terms-of-use 


\title{
Authors
}

Numbers in the index correspond to the last two digits of the six-digit citation identifier (CID) article numbering system used in Proceedings of SPIE. The first four digits reflect the volume number. Base 36 numbering is employed for the last two digits and indicates the order of articles within the volume. Numbers start with 00, 01, 02, 03, 04, 05, 06, 07, 08, 09, OA, OB...0Z, followed by 10-12, 20-2Z, etc.

\author{
Abautret, Johan, $1 R$ \\ Abdollahinia, Alireza, Ol, OL \\ Aho, Antti T., OS \\ Akimoto, Ryoichi, OC \\ Alias, Mohd S., OA \\ Al-Jabr, Ahmad A., OA \\ Alnahhas, Bayan A., OA \\ Anjum, Dalaver H., OA \\ Babazadeh, N., 06 \\ Badescu, S. C., 09 \\ Balzer, Jan C., 00 \\ Banyoudeh, Saddam, OI, OL \\ Baum, Philipp, $0 Q$ \\ Becker, Annette, $0 Q$ \\ Becker, S., 12 \\ Belenky, Gregory, 03, 0U \\ Bjelica, Marko, $0 Q$ \\ Blanchard, Romain, is \\ Boiko, D. L., $1 \mathrm{~J}$ \\ Botez, D., 13, 14 \\ Boyle, C., 13, 14 \\ Brenner, Carsten, 0N, 00 \\ Briggs, Ryan M., is \\ Brox, Olaf, 11 \\ Bugajski, M., IV \\ Bugge, Frank, 11 \\ Carpintero, Guillermo, 1R \\ Carras, Mathieu, IR \\ Chang, C.-C., 13 \\ Chang, TzU-Hsuan, OY \\ Childs, David T. D., 06, 1W, 21 \\ Cho, Sang June, OY \\ Chow, Weng W., 1E \\ Crump, P., $1 \mathrm{~L}$ \\ Czyszanowski, T., 1V \\ Decker, J., IL \\ d'Humières, B., 14 \\ Döpke, Benjamin, 0N, 00 \\ Dumitrescu, Mihail, OS \\ Earles, T., 13, 14 \\ Eisenstein, Gadi, OL, OQ \\ Ekterai, M., $1 \mathrm{~L}$ \\ Elafandy, Rami T., OA \\ Erbert, Götz, 05, 0N, 00, 1I, 1L \\ Erdelyi, Robertus, 21 \\ Esquivias, Ignacio, $1 \mathrm{~K}$ \\ Eyal, Ori, OL, OQ \\ Faugeron, Mickael, $1 \mathrm{~K}$ \\ Fazilleau, Yves, $1 \mathrm{U}$
}

Feng, Jijun, OC

Feng, T., 03

Fischer, M., 12

Forouhar, Siamak, OU, is

Fradet, Mathieu, OU, is

Frez, Clifford, OU, 1S

Fricke, Jörg, 05, 1I, $1 \mathrm{~L}$

Fujii, Takuro, 10

G. Tijero, José Manuel, $1 \mathrm{~K}$

Gao, Jun, OE

Garcia, M., $1 \mathrm{~J}$

Ghazal, O. M. S., 06

Gilles, Clément, 1R

Grahn, H. T., 1A

Groom, K. M., 06, IW

Hader, J., 09

Hasebe, Koichi, 10

Heinrich, W., 05

Hoffmann, Thomas, 05, OR

Höfling, S., 12

Hofmann, Martin R., ON, 00

Hogg, Richard A., 06, 1W, 21

Horstkemper, Heiko, ON

Hosoda, Takashi, 03, OU

Ivanov, Pavlo, 21

Johnson, S., 09

Kakitsuka, Takaaki, 10

Kamp, M., 12

Khamas, Salam, 21

Kim, Munho, OY

Kipshidze, G., 03

Kirch, J. D., 13, 14

Klehr, Andreas, 05, 0N, 00, OR

Knigge, S., $1 \mathrm{~L}$

Koch, S. W., 09

Koeth, J., 12

Krakowski, Michel, 1 J, $1 \mathrm{~K}$

Kryzhanovskaya, N. V., OJ

Kuc, M., IV

Kudashova, Yu. V., OJ

Kulagina, M. M., OJ

Kuramochi, Eiichi, 10

Lecomte, M., 1 J

Lee, Jaeseong, OY

Lei, D., 06

Lewicki, Rafał, $1 T$

Li, Biao, $1 T$

Li, Bin, OE

Li, Guangrui, 21 
Liero, Armin, 05, OR

Lindberg, D., III, 13, 14

Liu, Cheng, OF

Liu, Dong, OY

Livshits, D. A., OJ

Luo, Shiwen, OE

$\mathrm{Ma}$, Zhenqiang, OY

Maaßdorf, A., 1L

Maisons, Grégory, 1R

Maiwald, Martin, OT

Majid, Mohammed A., OA

Margoto, Éric, $1 \mathrm{U}$

Matsuo, Shinji, 10

Mawst, L. J., 13, 14

Maximov, M. V., OJ

Michael, Stephan, 1E

Moiseev, E. I., OJ

Moloney, J. V., 09

Müller, André, OT, 1 I

Nähle, L., 12

$\mathrm{Ng}$, Tien Khee, OA

Notomi, Masaya, 10

Ooi, Boon S., OA

Ooi, Yu Kee, OF

Orbe, Luis J., IR

Oubei, Hassan M., OA

Parillaud, O., $1 \mathrm{~J}$

Pérez-Serrano, Antonio, $1 \mathrm{~K}$

Pflügl, Christian, 1S

Pilny, Rouven H., ON, $0 \mathrm{O}$

Pohl, J., 05

Prziwarka, T., 05

Reithmaier, Johann Peter, OI, OL, OQ

Resneau, P., 1J

Rippien, Anna, OQ

Röben, B., 1A

Robert, Y., $1 \mathrm{~J}$

Rubel, O., 09

Salmi, Joel, OS

Sander, Stanley, OU

Sarma, Jayanta, 21

Sarzała, R. P., 1V

Schevermann, J., 12

Schnabel, Florian, $\mathrm{OL}, \mathrm{OQ}$

Schneider, Hans Christian, $1 \mathrm{E}$

Schrottke, L., $1 \mathrm{~A}$

Seo, Jung-Hun, OY

Shehata, Mohamed, OA

Shinya, Akihiko, 10

Shterengas, Leon, 03, 0U

Sichkovskyi, Vitalii, Ol, OL, OQ

Sigler, C., 14

Stevens, B. J., 06, 1W

Sumpf, Bernd, OR, OT, 11

Takeda, Koji, 10

Taylor, Richard J. E., 21

Tooley, I. G., IW

Tränkle, Günther, 0N, 0O, OR

Troshkov, S. I., 0J

Uusitalo, Topi, OS
Van Dijk, Frédéric, $1 \mathrm{~K}$

Viheriälä, Jukka, OS

Vilera, Mariafernanda, IK

Vinet, E., $1 \mathrm{~J}$

Virtanen, Heikki, OS

von Edlinger, M., 12

Vu, Thi N., OR

Wang, M., 03

Wang, Xinbing, $\mathrm{OE}$

Wang, Xudong, OY

Weih, R., 12

Wenzel, H., 05

Wienold, M., IA

Winterfeldt, M., $1 \mathrm{~L}$

Witinski, Mark, IT

Witzigmann, Bernd, $0 Q$

Wünsche, H.-J., 05

Wysocki, Gerard, $1 \mathrm{~T}$

Xia, Zhenyang, OY

Xiong, Dongsheng, $\mathrm{OE}$

Yin, Xin, OY

Yu, Anlan, OE

Zadiranov, YU. M., OJ

Zhang, Huilong, OY

Zhang, Jing, OF

Zhao, Deyin, OY

Zhao, Jun, OE

Zhou, Weidong, OY

Zhukov, A. E., OJ

Zubov, F. I., OJ

ZUo, Duluo, OE 


\section{Conference Committee}

Symposium Chairs

Jean-Emmanuel Broquin, IMEP-LAHC (France)

Shibin Jiang, AdValue Photonics, Inc. (United States)

Symposium Co-chairs

David L. Andrews, University of East Anglia (United Kingdom)

Alexei L. Glebov, OptiGrate Corporation (United States)

Program Track Chair

Klaus P. Streubel, OSRAM AG (Germany)

Conference Chairs

Alexey A. Belyanin, Texas A\&M University (United States)

Peter M. Smowton, Cardiff University (United Kingdom)

\section{Conference Program Committee}

Yasuhiko Arakawa, The University of Tokyo (Japan)

Mikhail A. Belkin, The University of Texas at Austin (United States)

Dan Botez, University of Wisconsin-Madison (United States)

Federico Capasso, Harvard School of Engineering and Applied Sciences (United States)

Gary A. Evans, Southern Methodist University (United States)

Michael Kneissl, Technische Universität Berlin (Germany)

Luke F. Lester, Virginia Polytechnic Institute and State University (United States)

Shinji Matsuo, NTT Photonics Laboratories (Japan)

Luke J. Mawst, University of Wisconsin-Madison (United States)

Jerry R. Meyer, U.S. Naval Research Laboratory (United States)

Roberto Paiella, Boston University (United States)

Richard V. Penty, University of Cambridge (United Kingdom)

Johann Peter Reithmaier, Universität Kassel (Germany)

Haisheng Rong, Intel Corporation (United States)

Gary M. Smith, MIT Lincoln Laboratory (United States)

Nelson Tansu, Lehigh University (United States)

Miriam Serena Vitiello, Consiglio Nazionale delle Ricerche (Italy)

Qi Jie Wang, Nanyang Technological University (Singapore) 
Session Chairs

1 Structure and Cavity Developments

Johann P. Reithmaier, Universität Kassel (Germany)

2 Materials Developments

Luke J. Mawst, University of Wisconsin-Madison (United States)

3 Blue/Green Emitters

Michael Kneissl, Technische Universität Berlin (Germany)

4 Quantum Dots

Samuel Shutts, Cardiff University (United Kingdom)

5 Cavity Effects and Mode-Locking

Luke F. Lester, Virginia Polytechnic Institute and State University (United States)

6 DFB and DBRs

Koji Takeda, NTT Device Technology Laboratories (Japan)

7 Lasers on Silicon

Haisheng Rong, Intel Corporation (United States)

8 Interband and Quantum Cascade Lasers

Jerry R. Meyer, U.S. Naval Research Laboratory (United States)

9 QCLs: Combs and Mode-Locking I

Sukhdeep Dhillon, Laboratoire Pierre Aigrain (France)

10 QCLs: Combs and Mode-Locking II

Gustavo F. Villares, ETH Zürich (Switzerland)

11 New Device Concepts

Andreas Wacker, Lund Universitet (Sweden)

12 High-Brightness Lasers

Gary M. Smith, MIT Lincoln Laboratory (United States)

13 Terahertz Quantum Cascade Lasers

Miriam Serena Vitiello, Consiglio Nazionale delle Ricerche (Italy)

14 QCLs: Cavities, Integration, and Applications

Dan Botez, University of Wisconsin-Madison (United States) 